\title{
Study on Cerebral Vascular Image of SD-OCT Via Orthogonal Matching Pursuit
}

\author{
Ping Xu and Ying-Jie Chang \\ College of Life Information Science and Instrument Engineering, Hangzhou Dianzi University, \\ Hangzhou, China \\ pingxu2011@163.com
}

Keywords: Cerebral Vascular Image, SD-OCT, Orthogonal Matching Pursuit

\begin{abstract}
This paper proposes the compressed method of Orthogonal Matching Pursuit to retrieve cerebral vascular image of spectral-domain optical coherence tomography (SD OCT). Discrete wavelet transform is used to sparse the signal. Original cerebral vascular image of spectral-domain optical coherence tomography is reconstructed with strong noise. The compressed method based on Orthogonal Matching Pursuit is applied to reconstruct the whole image. It is shown that the proposed CS method can achieve good PSNR performance compared to original method. Therefore, the proposed method is favorable for imaging of cerebral vascular image both SD OCT and SS-OCT.
\end{abstract}

\section{Introduction}

Optical coherence tomography (OCT) has been used widely in medical diagnostics and imaging for its noninvasive cross-sectional image with high resolution and fast imaging speed since its birth in 1991[1]. In recent years, due to its better sensitivity and faster imaging speed compared to time domain OCT (TD OCT), spectral-domain OCT (SD-OCT) has replaced conventional TD OCT in many medical applications[2]. SD-OCT uses a spectrometer and a linear array CCD to obtain the interferogram.

Since 2006, compressed sensing(CS)[4,5] has become a new research hotspot because of its potential ability to significantly reduce the amount of data acquisition in the field of mathematics and signal processing. CS has been applied in medical imaging such magnetic resonance imaging (MRI)[6] and photo-acoustic tomography [7, 8]. Until recent two or three years, CS has been introduced in OCT reconstuction[9,10]. Sparsity representation of the image data in a suitable transform domain largely determines the overall reconstruction quality[11]. The most frequently used transforms in CS OCT is wavelet transform and identity transform. Orthogonal Matching Pursuit(OMP) is easy and fast to reliably recover a signal with $\mathrm{m}$ nonzero entries in dimension $\mathrm{d}$ given $\mathrm{O}(\mathrm{m} \ln \mathrm{d})$ random linear measurements of the signal. In this study, we investigated the feasibility of applying OMP to retrieve cerebral vascular image of spectral-domain optical coherence tomography (SD OCT), which can achieve reasonable high quality images using partial data.

\section{Data Acquisition}

Figure 1 depicts the schematic diagram of the SD-OCT setup used in this study. We use a broadband superluminescent diode as light source that has a $\sim 80 \mathrm{~nm}$ effective bandwidth centered at $835 \mathrm{~nm}$. The spectral resolution of the spectrometer with a CCD (2048 pixels) is $0.0674 \mathrm{~nm}$.The measured axial resolution is $6.8 \mu \mathrm{m}$. The speed of A-scan is $29 \mathrm{kHz}$ and the maximum imaging depth is $2.56 \mathrm{~mm}$ with maximum signal-to-noise ratio $115 \mathrm{~dB}$.

In this SD-OCT system, we get the interferograms of a total of $256 \mathrm{x}$-axes. The signal can be represented by the matrix of 2048 rows and 256 columns. The reconstructed image can be obtained by the following steps. Firstly, the mean value of each row is calculated, then each element subtracts the mean value of its row. Secondly, the FFT transform and FFT shift transform are used to get the value of intermediate result sequentially. Thirdly, after getting the absolute value, log operator is used to calculate the result. Fourthly, mean filter is used to process the result, then extraction operator to 
reconstruct image with 511 rows and 512 columns. At last, a row with all zeros is added to the end row to get the ultimate reconstructed image with 512 rows and 512 columns.

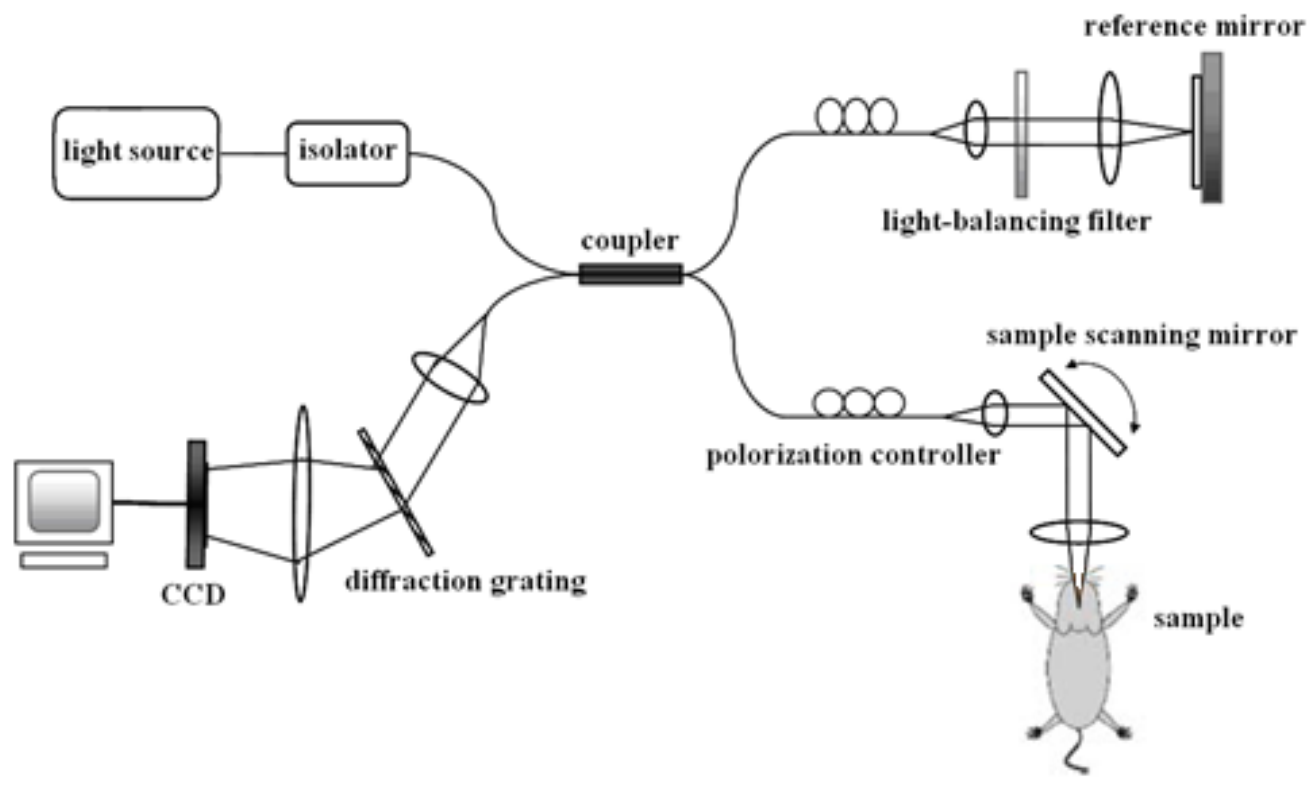

Fig. 1 Schematic of the spectral domain OCT system used to monitor rat's cerebral vascular image.

\section{The OMP Algorithm}

This section describes the OMP algorithm in detail, which is a greedy algorithm for signal recovery. Suppose that $\mathrm{x}$ is an arbitrary $\mathrm{M}$-sparse signal in $\mathrm{R}^{\mathrm{N} \times 1}$, that is to say that there are $\mathrm{M}$ elements are non-zero in itself or in its transform domain $\Psi$. Let $\left\{\mathrm{y}_{1}, \ldots, \mathrm{y}_{\mathrm{M}}\right\}$ be a family of $\mathrm{M}$ measurement vectors of $\mathrm{x}$ :

$$
y_{i}=<\phi_{i}, x>, i=1, \cdots, M, M<N
$$

Where $\phi 1, \phi 2, \ldots, \phi_{\mathrm{M}}$ are the measurement vectors and form the measurement matrix $\Phi$ which is irrelevant to $\Psi$.

Let $P=\Phi \Psi$, $\mathrm{t}$ is the iterations. The OMP algorithm can be described as follows:

- Step 1: Initialize the residual $\mathrm{r} 0=\mathrm{y}$, the estimated value $\hat{y}=0, \hat{y} \in R^{N}$ and $\mathrm{t}=1$;

- Step 2: Find the most relevant column index in $P$ :

$$
k_{t}=\arg \max _{i=1, \ldots, N}<r_{t-1}, p_{i}>
$$

- Step 3: Set $\lambda_{t}=\lambda_{t-1} \cup\left\{k_{t}\right\}$

- Step 4: Get the orthogonal projector $P_{t}$ on span $\left\{p_{k}: k \in \lambda_{t}\right\}$

- Step 5: Determine the residual as follows:

$$
q_{t}=P_{t} y \hat{\lambda}
$$

- Step 6: Set $t=t+1$, and return to Step 2 if $t<M$

- Step 7: The optimal estimate value $\hat{y}$ for the signal $y$ has non-zero indices list in $\lambda_{M}$. The values of the estimate can be calculated as:

$$
q_{M}=\sum_{k \in \lambda_{M}} \hat{y}_{k} p_{k}
$$




\section{Experimental Results}

In this session, the OMP algorithm is used to reconstruct the SD-OCT image with M of 300 . Discrete wavelet transform is used to sparse the input image. Fig 1 shows the structural image of cerebral vascular image with different methods. Figure2.(a) is the original reconstructed image. Figure2.(b) shows the resultant image using the proposed CS method with $M$ of 300. Figure2.(c) shows the local image of the original reconstructed cerebral vascular. Figure2.(d) shows the local reconstructed cerebral vascular image using the proposed CS method with M of 300. Fig 2 shows another structural image of cerebral vascular image with different methods. Figure3.(a) is another original reconstructed image. Figure3.(b) shows the resultant image using the proposed CS method with $\mathrm{M}$ of 300. Figure3.(c) shows the local image of another original reconstructed cerebral vascular. Figure3.(d) shows the local reconstructed cerebral vascular image using the proposed CS method. Although original SD-OCT images show strong noise, the SNR advantage of can be clearly observed compared to the original method. It is worth mentioning that the proposed CS method does not show down-sampling coherent alising. The above results show that the proposed compressed sensing method can achieve high SNR structural image of cerebral vascular image with partial data.

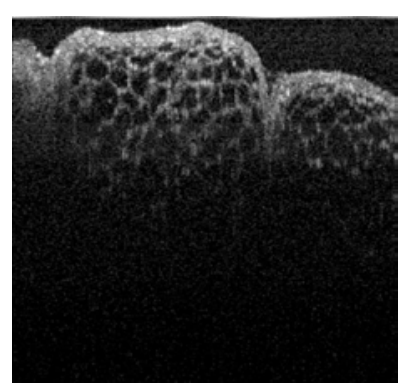

(a)

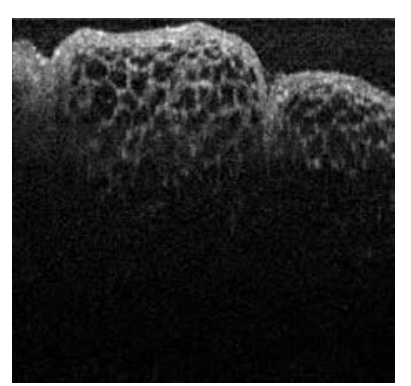

(b)

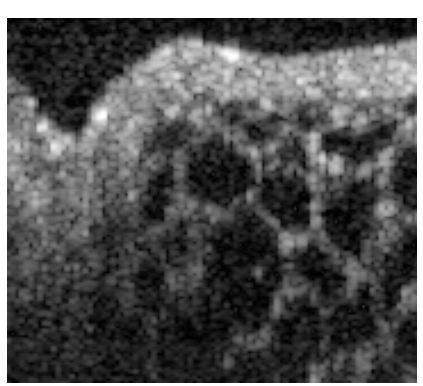

(c)

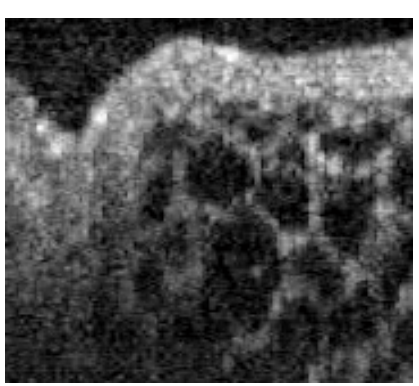

(d)

Fig2 shows OCT structural images of cerebral vascular image with different methods.

(a) original method (b) reconstructed image of the proposed CS method. (c) local image of the original reconstructed image. (d) local image of the reconstructed image of the proposed CS method.

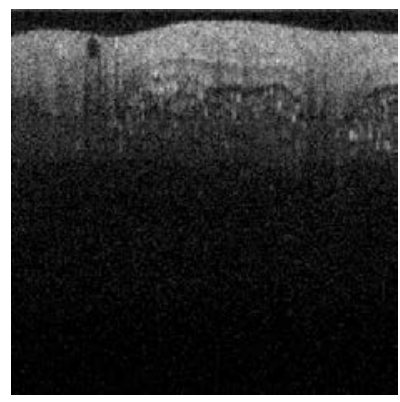

(a)

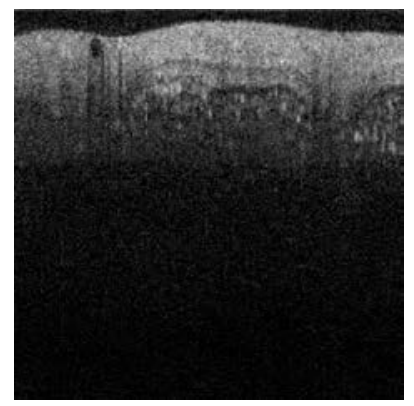

(b)

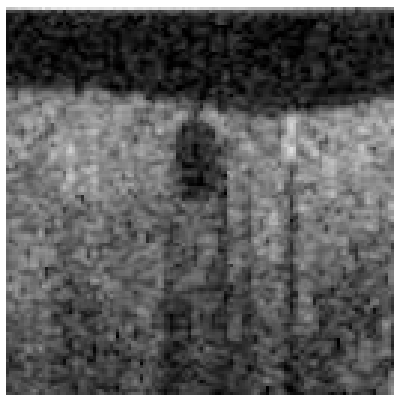

(c)

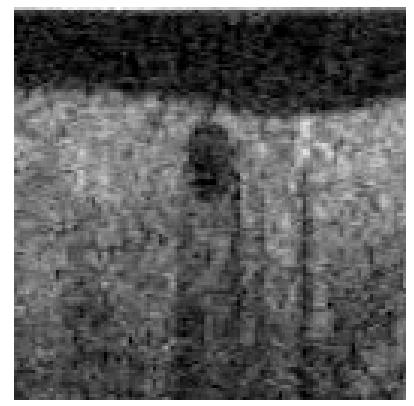

(d)

Figure3. shows another OCT structural images of cerebral vascular image with different methods.

(a) original method (b) reconstructed image of the proposed CS method. (c) local image of the original reconstructed image. (d) local image of the reconstructed image of the proposed CS method.

\section{Conclusion}

In conclusion, a novel compressed sensing method of Orthogonal Matching Pursuit is developed to reconstruct the structural OCT images of cerebral vascular image. It is shown that the proposed CS method can achieve good PSNR performance for the structural OCT images of cerebral vascular image with strong noise. The proposed OMP method is favorable for imaging of cerebral vascular image both SD OCT and SS-OCT. 


\section{ACKNOWLEDGMENTS}

This research was sponsored by the National Science Foundation of China (NSFC), grants 61205200, and Zhejiang Provincial Natural Science Foundation of China(ZJNSF), grants LY12F01005.

\section{References}

[1] D. Huang, E.A. Swanson, C.P. Lin, J.S. Schuman, W.G. Stinson, W. Chang, M.R. Hee, T. Flotte, K. Gregory, C.A. Puliafito, J.G. Fujimoto, Science, 254, 1178(1991).

[2] P.E. Andersen, L. Thrane, H.T. Yura, A. Tycho, T.M. Jørgensen, M.H. Frosz, Phys. Med. Biol. 49, 1307(2004).

[3] Xuan Liu and Jin U. Kang,Sparse OCT: Optimizing compressed sensing in spectral domain optical coherence tomography, PMC 2012 May 17.

[4] E. J. Cand `es, J. K. Romberg, and T. Tao, "Stable signal recovery from incomplete and inaccurate measurements”, Communications on Pure and Applied Mathematics, 59, 1207 (2006).

[5] D. L. Donoho, “Compressed sensing” , IEEE Transactions on Information Theory, 52, 1289-1306 (2006).

[6] M. Lustig, D. Donoho, and J. M. Pauly, "Sparse mri: The application of compressed sensing for rapid mr imaging,” Magnetic Resonance in Medicine, 58, 1182-1195 (2007).

[7] J. Provost and F. Lesage, "The application of compressed sensing for photo-acoustic tomography.” IEEE Transactions on Medical Imaging 28, 585-594 (2008).

[8] Z. Guo, C. Li, L. Song, and L. V. Wang, "Compressed sensing in photoacoustic tomography in vivo,” Journal of Biomedical Optics 15, 021311 (2010).

[9]. X. Liu and J. U. Kang, Sparse OCT: Optimizing compressed sensing in spectral domain optical coherence tomography, Opt. Express 18, 22010 (2010).

[10] Ning Zhang, Tiancheng Huo, Chengming Wang, Tianyuan Chen, Jing-gao Zheng, and Ping Xue, Compressed sensing with linear-in-wavenumber sampling in spectral-domain optical coherence tomography, Optics Letters, 37,3075-3077, 2012.8.

[11] Wei Chen, Zhaoyang Jin, Feng Liu, Yiping P. Du, Application of SVD-based sparsity in Compressed Sensing Susceptibility Weighted Imaging, 2012 5th International Conference on BioMedical Engineering and Informatics (BMEI 2012), 311-314.

[12] M. Hong, Y. Yu, H. Wang, F. Liu and S. Crozier, "Compressed sensing MRI with singular value decoposition- based sparsity basis”, Phy. Med, Biol.56, 6311-6325(2011).

[13] T. T.Cai and L.Wang, “Orthogonal Matching Pursuit for Sparse Signal Recovery With Noise”, IEEE Transactions on Information Theory, 57, 4680-4687(2011.7).

[14] Laura Rebollo-Neira and David Lowe, “Optimized Orthogonal Matching Pursuit Approach”, IEEE Signal Processing Letters, 9, 137-140(2002.4). 